\title{
Effects of magnetohydrodynamic waves in Jovian decametric emission
}

\author{
O. V. Arkhypov ${ }^{1}$ and H. O. Rucker ${ }^{2}$ \\ 1 Institute of Radio Astronomy, National Academy of Sciences of Ukraine, Chervonopraporna 4, 61002 Kharkiv, Ukraine \\ e-mail: rai@ira.kharkov.ua \\ 2 Space Research Institute, Austrian Academy of Sciences, Schmiedlstrasse 6, 8042 Graz, Austria \\ e-mail: rucker@oeaw.ac.at
}

Received 12 June 2007 / Accepted 15 August 2007

ABSTRACT

\begin{abstract}
The flux variability of Jovian decametric radio emission (DAM) is analyzed to search for its possible modulations by magnetohydrodynamic waves of ultra-low frequencies (ULF). The ULF modulating waves are found in DAM dynamic spectra as a moving pattern (moire) on the background of Jovian millisecond radio bursts (S-bursts). Their frequency drift ( $\sim 55 \mathrm{MHz} / \mathrm{s})$ corresponds to the wave motion to Jupiter with Alfvén velocity $\left(\sim 4 \times 10^{4} \mathrm{~km} \mathrm{~s}^{-1}\right)$. There are whistler-like drifting details in the dynamic power spectra of ULF variations of S-burst emission flux at fixed radio frequency. Their frequency drift rates are consistent with whistlers that are dispersed mainly in the Io torus. Formally our analysis of DAM reveals the specific 2s-modulation of S-burst emission corresponding to the known magnetic pulsations near gyrofrequencies of heaviest ions $\left(\mathrm{SO}_{2}^{+}\right.$and $\left.\mathrm{SO}^{+}\right)$in the Io torus.
\end{abstract}

Key words. planets and satellites: individual: Jupiter - radio continuum: solar system - magnetohydrodynamics (MHD) - waves plasmas - instabilities

\section{Introduction}

It is widely accepted that waves of ultra-low frequencies (ULF) can be studied only in situ, with space probes. Also other emissions from Jupiter like hectometric (HOM) and kilometric (KOM) radiations are only observed by satellite or spacecraft because of the Earth's ionospheric cut-off (Zarka 1998). Only decametric and decimetric radio emissions of Jovian magnetosphere are observable from the Earth. However, modern theories connect the planetary radio emission with ULF Alfvén waves, which accelerate electrons and stimulate plasma instabilities with electromagnetic wave generation near Jupiter and other planets (e.g.: Belcher 1987; Ergun et al. 2006; Su et al. 2006; Zarka 1998).

Analogously, Hanasz et al. (2006) find in the case of the Earth that Alfvénic vibrations of magnetic field lines are able to drive pulsations of terrestrial kilometric radiation. It follows from correlated observations made by the Interball-2 spacecraft and the IMAGE magnetometer array that such radio pulsations often occur (in 39 out of 61 cases) simultaneously with field line resonances and at frequencies near to those of vibrations.

Apparently, such an approach could open the way to earthbased studies of ULF oscillations in the Jovian magnetosphere. Some signs of DAM modulations by a standing Alfvén wave have indeed been found recently in the Jovian ionosphere as the pattern of S-burst bands in the dynamic spectrum (Arkhypov \& Rucker 2006). Another modulation effect is the system of periodic peaks (the eigenfrequencies of the Alfvén resonator) in the power spectra of ULF oscillations of S-burst emission flux, which is recorded at fixed radio frequency.

Our purpose is to continue that search for ULF effects in DAM, which could be connected with propagating magnetohydrodynamic waves near Jupiter.

\section{Propagating wave of modulation}

According to the standard model (e.g.: Zarka 1998), the Io-related radio emission is generated on the active Jovian magnetic line, which are disturbed by the Io satellite (Fig. 1). The observed frequency $f$ is approximately equal to the local electron cyclotron frequency $f_{c e}$ at the point of the active line from which it is emitted. Hence, if a compact radio source is moving along such magnetic line away from Jupiter, a narrow emission strip (S-burst) is drifting to low frequency in the dynamic spectrum (Ellis 1982; Hess et al. 2007). An ULF disturbance of this motion by hydromagnetic waves could modulate the emission flux along its trajectory in spectrum and bend the S-burst structure (see the review in Arkhypov \& Rucker 2006).

As a result of this, ULF modulating waves could be found on the DAM dynamic spectra as a moving pattern (moire) on the background of dense train of S-bursts. Such a moire is found on the dynamic spectrum of the Io-B S-storm of 1988 November 12 , 22:12 UT (Riihimaa 1991, Fig. 14). For visualization of the hidden pattern, this spectrum (Fig. 2a) has been smoothed to remove individual S-bursts and to retain larger details. These details are contrasted into the black-white scheme (Fig. 2b). As a result of this processing, the system of bands with the positive frequency drift of their medians is visible in Fig. 2c.

The visualized pattern differs markedly from well-known modulation lanes. Its period is $0.17 \pm 0.04 \mathrm{~s}$, while modulation lanes have a time scale from $\sim 1 \mathrm{~s}$ (Imai et al. 1997) to $\sim 15 \mathrm{~s}$ (Arkhipov 2004). There is a large discrepancy in the frequency drift rate: $\sim 50 \mathrm{MHz} / \mathrm{s}$ contrary to $\sim 0.1 \mathrm{MHz} / \mathrm{s}$ for lane modulations (Imai et al. 1997; Riihimaa 1993). At $168^{\circ}$ elongation, the interplanetary scintillations of DAM are too long $(\sim 10 \mathrm{~s}$; Genova \& Leblanc 1981). The DAM modulations from the terrestrial ionosphere have too long a time scale $(\sim 40 \mathrm{~s})$ and a slow 


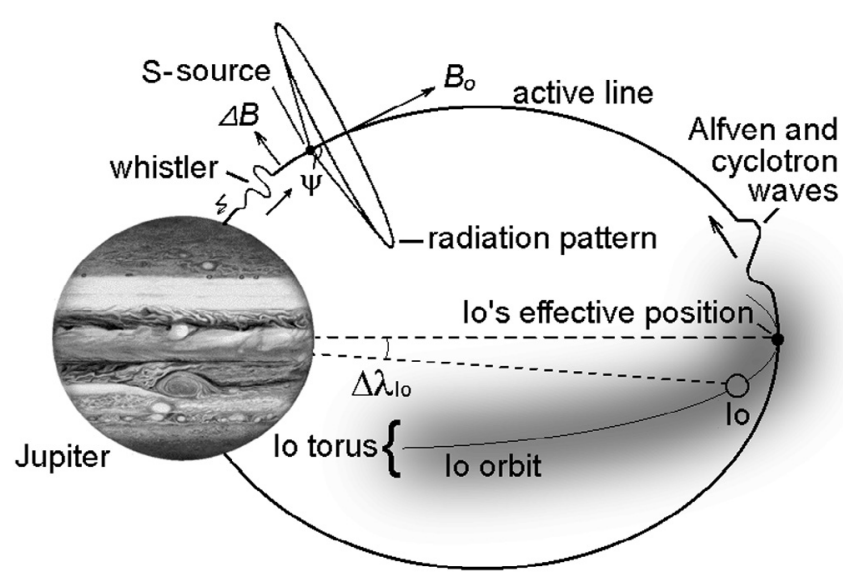

Fig. 1. Scheme of generation and modulation of the Jovian decametric emission (not to scale).

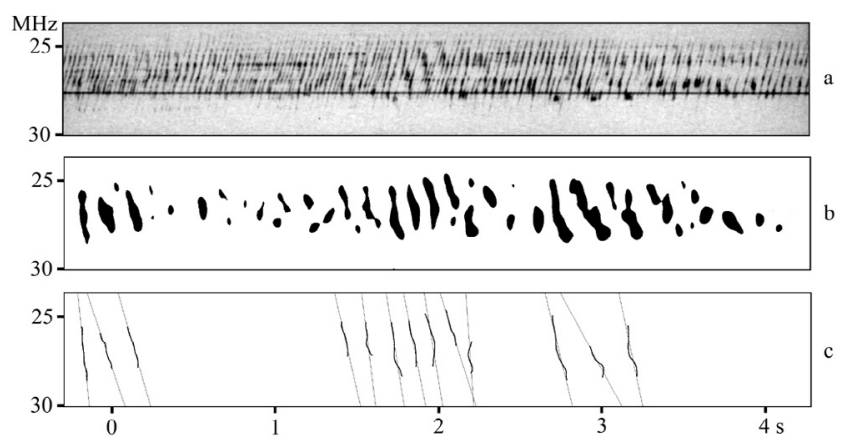

Fig. 2. ULF wave modulation seen as the regular pattern (moire) on the background of S-train: a) the fragment of dynamical spectrum of November 12, 1988 (Riihimaa 1991, Fig. 14); b) the visualized moire spots from wave packets that propagate towards the higher gyrofrequencies, i.e. towards Jupiter; c) estimation of moire drift with the linear approximation (thin lines) of longest spot medians (solid curves).

frequency drift ( $\sim 0.02 \mathrm{MHz} / \mathrm{s}$; Genova et al. 1981). Apparently, Fig. 2 demonstrates the unknown type of DAM modulation.

The linear approximation of the band medians (Fig. 2c) gives the mean drift rate of $\mathrm{d} f / \mathrm{d} t=52.3 \pm 8.5 \mathrm{MHz} / \mathrm{s}$. The gradient of electron gyrofrequency along the active magnetic line $\mathrm{d} f_{c e} / \mathrm{d} x=$ $1.29 \mathrm{kHz} / \mathrm{km}$ is estimated by means of the VIP4 magnetic model (Connerney 1998) for a northern source (RH polarization; Riihimaa 1991), the spectrum time, radiation frequencies of $25-29 \mathrm{MHz}$, and the lead angle of $39^{\circ}$ for Io-B storms (found by the modulation lane method; Arkhipov 2003; Arkhypov \& Rucker 2007). Hence, the pattern's velocity along the magnetic field is $\mathrm{d} x / \mathrm{d} t \approx(\mathrm{d} f / \mathrm{d} t)\left(\mathrm{d} f_{c e} / \mathrm{d} x\right)^{-1}=40500 \pm 6600 \mathrm{~km} \mathrm{~s}^{-1}$. Analogous processing of another spectrum (1988 November 12, 21:59 UT; Riihimaa 1991, Fig. 3a) gives the pattern drift of $58.4 \pm 5.8 \mathrm{MHz} / \mathrm{s}$ and the velocity of $44900 \pm 4500 \mathrm{~km} \mathrm{~s}^{-1}$.

These estimations of modulation velocity are on the order of the Alfvén velocity $\left(6 \times 10^{4} \mathrm{~km} \mathrm{~s}^{-1}\right.$ at $\left.22 \mathrm{MHz}\right)$ that follows from the analysis of DAM modulation by a standing Alfvén wave in the Jovian ionospheric resonator (Arkhypov \& Rucker 2006). This result agrees with the electron number density of $N_{\mathrm{e}} \sim 10^{5} \mathrm{~cm}^{-3}$ just above the main ionosphere maximum during daylight hours, as it was found with the radio occultation curves (Strobel \& Atreya 1983; Yelle \& Miller 2004). In this case, the Alfvén velocity is $V_{A}=B / \sqrt{\mu_{\mathrm{o}} m_{i} n_{i}}=6 \times 10^{4} \mathrm{~km} \mathrm{~s}^{-1}$, where $B=9.6 \times 10^{-4} \mathrm{~T}$ is the magnetic induction in the radio source

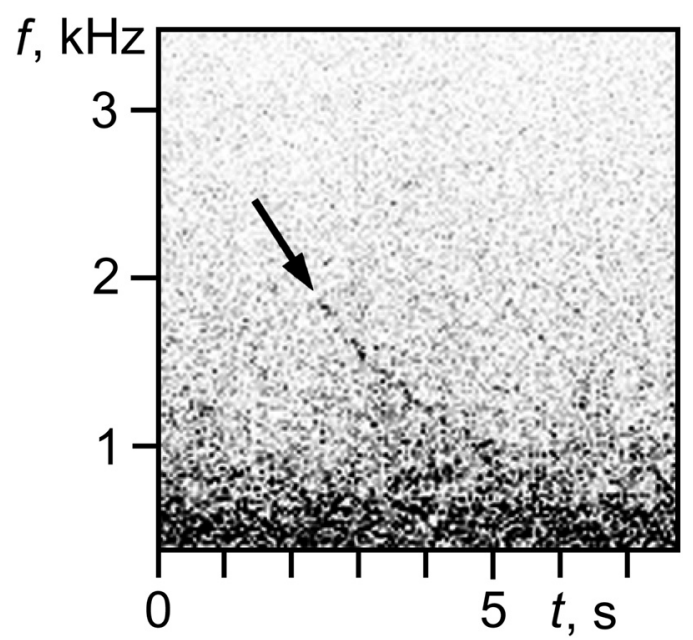

Fig. 3. Jovian whistler example is recorded in the Io torus by the Voyager's plasma wave instrument. This signal is extracted by Fourier transform from the demonstration audio-file (http://www.astrosurf.org/luxorion/Radio/ voyager-jupiter-lightnings.wav).

according to the $f=f_{c e}$ approximation; $\mu_{\mathrm{o}}=1.26 \times 10^{-6} \mathrm{H} / \mathrm{m}$ is the magnetic constant; $m_{i}=1.66 \times 10^{-27} \mathrm{~kg}$ is the ion (proton) mass; $n_{i}=n_{e}=10^{5} \mathrm{~cm}^{-3}=10^{11} \mathrm{~m}^{-3}$ is the ion number density. Hydrogen is about $90 \%$ of the Jupiter composition.

The second characteristic velocity for ULF waves in a cold ionospheric plasma is the sound speed of $C_{\mathrm{s}}=\sqrt{k_{\mathrm{b}} T / m_{i}} \sim$ $3 \mathrm{~km} \mathrm{~s}^{-1}$ (Nicholson 1983), where $k_{\mathrm{b}}=1.38 \times 10^{-23} \mathrm{~J} / \mathrm{deg}$ is the Boltzmann constant, and $T \sim 1000 \mathrm{~K}$ is the exospheric temperature (Yelle \& Miller 2004). Hence, only Alfvén velocity is commensurable with the moire drift rate. Therefore, the found moire in the DAM spectra indeed corresponds to the ULF modulating waves with quasi-Alfvénic velocity.

\section{Possible whistler effect}

Whistlers from lightning discharges in Jupiter's atmosphere (Fig. 3) have been discovered by the Voyager-1 space probe (Gurnett et al. 1979). These transverse waves oscillate the magnetic vector in the radio source. As this vector is an axis of DAM radiation pattern in the form of hollow cone (Fig. 1), such oscillations could turn the DAM beam away from or to the Earth. This modulation effect would be especially observable with narrow $\left(0.5^{\circ}\right.$ to $2^{\circ}$; Ellis 1982 ; Zarka 1998$)$ radio beams of individual S-bursts.

To obtain the observable oscillations of $\Delta \alpha \sim 1^{\circ}$ in the radio beam direction, the magnetic disturbance must be on the order of $\Delta B \sim \Delta \alpha B_{\mathrm{o}} \sim 1.2 \times 10^{-5} \mathrm{~T}$, where $B_{\mathrm{o}}=f_{\mathrm{o}} / K ; f_{\mathrm{o}}=20 \mathrm{MHz}$ is the frequency of radio emission about local $f_{c e} ; K=2.8 \times$ $10^{10} \mathrm{~Hz} / \mathrm{T}$ is a conversion coefficient between $f_{c e}$ and $B_{0}$. Then the energy of the modulation wave is (Kuzmichev 1989)

$W=\mu_{\mathrm{o}} \mu \Delta H^{2} s V_{\mathrm{g}} \Delta t \cos \gamma$,

where $\Delta H=\Delta B / \mu_{\mathrm{o}} \mu$ is the magnetic field strength; $\mu_{\mathrm{o}}=$ $1.26 \times 10^{-6} \mathrm{H} / \mathrm{m}$ is the vacuum permeability; $\mu=1$ is the magnetic permeability of the medium; $V_{\mathrm{g}} \sim c=3 \times 10^{8} \mathrm{~m} \mathrm{~s}^{-1}$ is the velocity of modulation wave in the radio source (about electromagnetic constant $c) ; \Delta t \sim 10^{-3} \mathrm{~s}$ is the typical duration of a Jovian lightning discharge (Farrell et al. 1999); $\gamma=0$ is the angle between the group velocity $V_{\mathrm{g}}$ and the normal to the properly oriented area $s$, illuminated by the wave. The minimal estimation 
of necessary whistler energy $\left(W \sim 3 \times 10^{12} \mathrm{~J}\right)$ could be found for waves that are channeled in field-aligned inhomogeneities with $s \sim \lambda^{2} \sim 9 \times 10^{10} \mathrm{~m}^{2}$ (where $\lambda=c / f$ is the wavelength at $f=1 / \Delta t \sim 1 \mathrm{kHz}$ ). This is about the average energy of observed Jovian lightning discharges $\left(1.7 \times 10^{12} \mathrm{~J}\right.$; Borucki et al. 1982). However, terrestrial superbolts have the energy about 500 times that of average lightning (Borucki et al. 1982). Therefore, the DAM modulation by Jovian superbolts is not excluded.

We searched for whistler-like signals in the collection of DAM records in the Space Research Institute (Austrian Academy of Sciences, Graz) and with the site of NASA's Radio Jove Project (http://jovearchive.gsfc.nasa.gov/). We calculated an analog of a classic dynamic spectrum where the relative intensity is shown versus the frequency and the time. However, it was done at ULF frequencies, and ULF fluctuations of S-burst intensity at fixed radio frequency were extracted from dynamic spectra of DAM or from *.wav-files. Then the squared harmonics of Fourier transform from the intensity variations was calculated at successive time intervals:

$C_{m}^{2}=A_{m}^{2}+B_{m}^{2}$

$A_{m}=\frac{1}{N} \sum_{i=1}^{N} F_{i} \cos \frac{2 \pi m i}{N}$,

$B_{m}=\frac{1}{N} \sum_{i=1}^{N} F_{i} \sin \frac{2 \pi m i}{N}$,

where $m$ is the harmonic number; $N$ is the number of all intensity readings $\left(F_{i}\right)$ in the analyzed time interval; $i$ is the intensity reading number. The obtained power spectrum is normalized with the amplitude of first harmonic $C_{1}$, and the parameter $\frac{C_{m}^{2}}{C_{1}^{2}}$ is coded in 256 colors as a vertical line on the dynamic spectra. To visualize delicate structures, the resulting 2D spectra were contrasted in the black-white schemes.

Usually such schemes show maximal spectral power (black) at low frequencies. Characteristic vertical details on the spectra are a result of the high variability of S-burst emission. There are horizontal diffusive spots that reflect periodicity of S-bursts. Occasionally some delicate drifting structures are distinguishable.

Examples of dynamic ULF spectra with features resembling the curved whistler with negative frequency drift in Fig. 3 are shown in Fig. 4. It is reasonable to describe these discoveries not as evidence, but rather as a demonstration of the possibility and guiding line for future studies.

As Jovian whistlers travel approximately along magnetic field lines (Gurnett et al. 1979, 1981), the radio source interacts with waves that intersect Io's torus. The main part of time delay of the whistler is formed just in the torus. A well-known characteristic of whistlers at low frequencies is that the arrival time $(t)$ at a specific frequency $(f)$ is proportional to $f^{-1 / 2}$ (Gurnett et al. 1981):

$t=D f^{-1 / 2}+t_{\mathrm{o}}$,

where $D$ is a constant called the dispersion, and $t_{\mathrm{o}}$ a time constant. Hence, the frequency drift is

$\mathrm{d} f / \mathrm{d} t=-2 \frac{f^{3 / 2}}{D}=-3.8 \mathrm{~Hz} / \mathrm{s}$,

where $f=80 \mathrm{~Hz} ; D=377 \mathrm{~s} \mathrm{~Hz}^{1 / 2}$ is an average dispersion of the whistler, which crossed the Io torus according to the

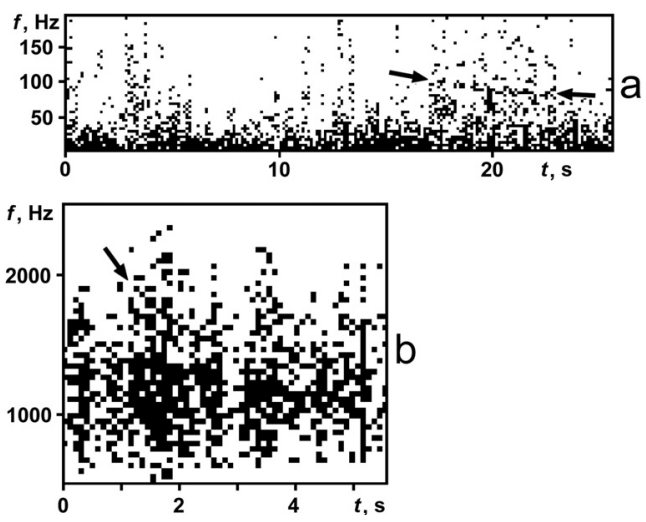

Fig. 4. Examples of whistler-like features with low drift rate (arrowed) in the records of S-burst emission at fixed frequency: a) $23.8 \mathrm{MHz}$ (2000 March 30, 11:44 UT; UTR-2 radio telescope, IRA, Kharkiv); b) $20.1 \mathrm{MHz}$ (2000 October 18, 08:07 UT; Ashcraft 2000). These dynamic power spectra of S-burst emission fluctuations are constructed with Fourier transform and the threshold filter.

Voyager 1 data (Gurnett et al. 1981; Table 4). The measured frequency drift for the strip at $f \approx 80 \mathrm{~Hz}$ is $-4.9 \mathrm{~Hz} / \mathrm{s}$ in Fig. 4a. There is only $29 \%$ difference with the prediction.

Another example of a whistler-like detail (Fig. 4b) is found in the audio record of S-bursts at $20.1 \mathrm{MHz} 2000$ October 18 (Ashcraft 2000). The observed drift rate is $-293 \pm 24 \mathrm{~Hz} / \mathrm{s}$ at $f \approx 1660 \mathrm{~Hz}$, and the predicted drift from (6) is $-359 \mathrm{~Hz} / \mathrm{s}$. There is only a $23 \%$-difference.

As the drifts in Fig. 4 formally correspond to those whistlers that crossed the Io torus, any future search for such modulation seems a promising task for UTR-2 and LOFAR radio telescopes with modern recording technology (Lecacheux et al. 2004).

\section{Search for ion cyclotron waves from lo torus}

Ion cyclotron waves can stimulate terrestrial kilometric radiation (TKR) by modulation of the electron phase space density (Mutel et al. 2007; Xin \& Menietti 2007). As TKR and DAM have similar origin (cyclotron maser instability of electrons in the polar magnetosphere; Zarka 1998), the search for DAM modulation by ion cyclotron waves seems reasonable.

The ion cyclotron waves with frequencies near the equatorial gyrofrequencies of $\mathrm{SO}_{2}^{+}$and $\mathrm{SO}^{+}$have been discovered with the Galileo space probe near the Io wake, in the Io plasma torus around Jupiter (Kivelson et al. 1996; Russell et al. 2003). It is very important that the amplitude of $\mathrm{SO}_{2}^{+} / \mathrm{SO}^{+}$cyclotron waves $(250 \mathrm{nT})$ is comparable to the transverse Io's disturbance of the Jovian magnetic field (300 nT; Kivelson et al. 1996). As the Io's disturbances (Alfvén wings) are considered a stimulus of Io-related DAM, L-emission (Bagenal \& Leblanc 1988) as well as S-bursts (Zarka 1998; Ergun et al. 2006), the ion cyclotron waves could have an influence on the Jovian radio emission, too. It has been argued that Alfvén waves accelerate electrons by parallel electric fields at Io (Zarka 1998) or at S-burst sources (Ergun et al. 2006) and stimulate plasma instabilities with electromagnetic wave generation near Jupiter.

The cyclotron wave near the gyrofrequency of heaviest ions propagates along the magnetic line from the Io wake towards Jupiter without gyroresonance absorption by light-weight ions. Near the planet, the local cyclotron frequency of ions (mainly protons) exceeds the frequency of ULF wave a lot, which is Alfvénic in the region of DAM sources. Such an Alfvén wave could modulate the acceleration of electrons (hence the DAM 


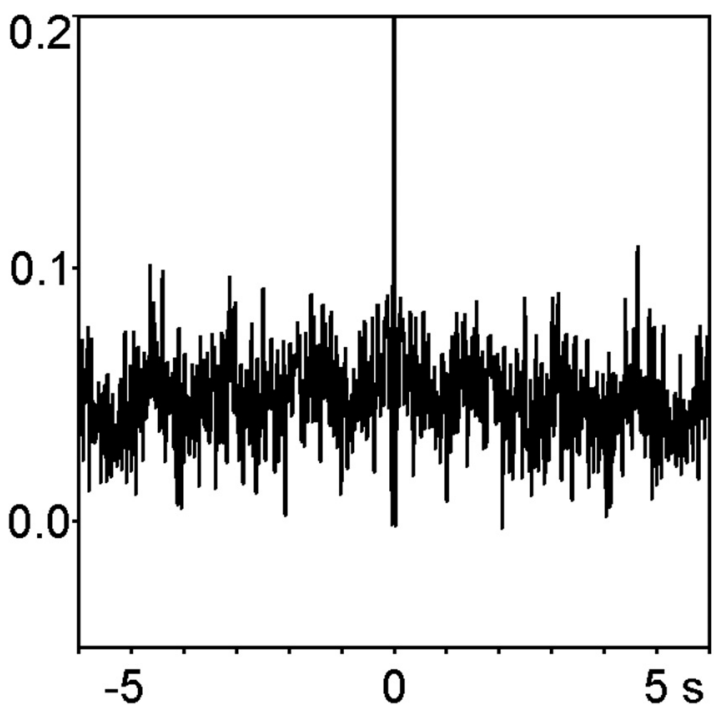

Fig. 5. The autocorrelation of the S-burst emission intensity in the record of 2002 August 9 (10:30 UT; $21.1 \mathrm{MHz}$; UTR-2 radio telescope, IRA, Kharkiv) showing the existence of a dominating wave with $1.5-2 \mathrm{~s}$ period, which corresponds to the $\mathrm{SO}_{2}^{+} / \mathrm{SO}^{+}$ion cyclotron waves found in the Io plasma torus (Kivelson et al. 1996; Russell et al. 2003).

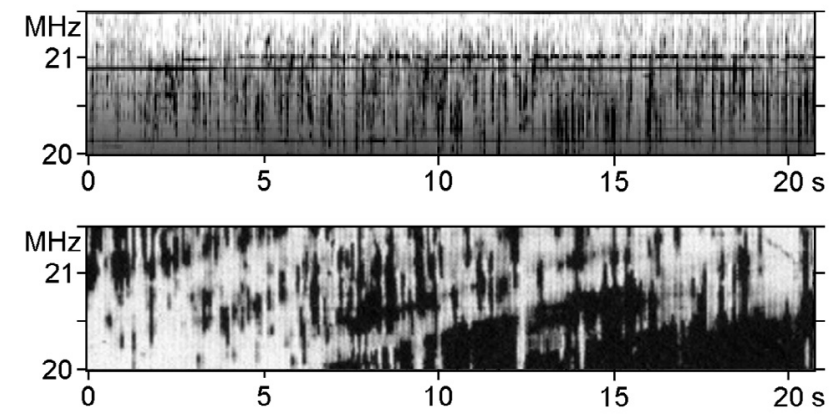

Fig. 6. The dynamic radio spectrum of S-burst emission (above). It is processed in Fig. 5, and has no regular modulation lanes such as of drifting bands on the bottom spectra of 1987 October 24, 00:11 UT (Riihimaa 1993, Fig. 34) with similar central meridian longitude and Io position on its orbit.

generation) or bring the DAM source into an oscillatory motion. Hence, the ion cyclotron frequency of Io torus may be imprinted in DAM flux variations.

The frequency $\sim 0.5 \mathrm{~Hz}$ of heaviest $\mathrm{SO}_{2}^{+} / \mathrm{SO}^{+}$ions dominates in the magnetometer data of Galileo (Kivelson et al. 1996; Russell et al. 2003). In this context, the 2 s-periodicity in the autocorrelation function of the S-burst emission record seems very interesting (Fig. 5). We used the dynamic spectrum of the Io-B S-storm, which was recorded on 2002 August 9 at 10:30 UT in the frequency range from $17.3 \mathrm{MHz}$ to $29.8 \mathrm{MHz}$ with UTR-2 radio telescope of the Institute of Radio Astronomy (Nat. Acad. Sc. of Ukraine, Kharkiv). The measurements at 21.1 MHz were selected for the autocorrelation analysis because of both the absence of interference and the maximal duration of S-burst emission. The L-component was undetected. The 9983 readings of relative intensity of S-burst emission during $59.706 \mathrm{~s}$ are processed in Fig. 5.

Apparently, this 2s-periodicity is not an effect from the modulation lanes, which are formed by DAM scattering on fieldaligned inhomogeneities mainly in the Io plasma torus (Imai et al. 1997; Arkhypov \& Rucker 2007). Figure 6 shows that
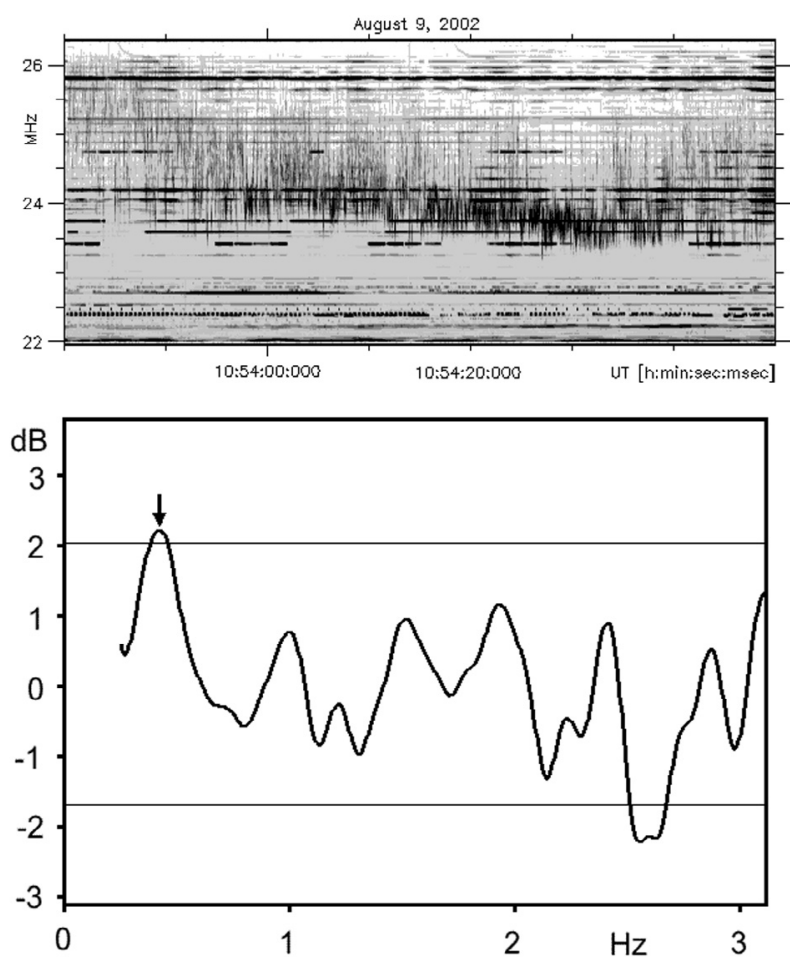

Fig. 7. The oscillations of the S-band recorded at the Nançay Radio Observatory on 2002 August 9 (above). The corresponding Fourier power spectrum (bottom) of time variations of S-burst intensity at $24.3 \mathrm{MHz}$ has a peak at $0.43 \mathrm{~Hz}$ with 0.99 significance. The interval of 0.98 -probability for spectral estimations (between horizontal lines) is calculated with a standard $\chi^{2}$-distribution.

no regular modulation lanes are seen on that dynamic spectrum. Moreover, interplanetary scintillations have too long a time scale of $>10 \mathrm{~s}$ at that Jupiter elongation of $15^{\circ}$ from the Sun (Genova \& Leblanc 1981) for explaining the 2 s-modulation. Independent confirmation could be found in the data of Nançay Radio Observatory (Fig. 7). There is a significant (0.99) peak in the Fourier spectrum at $0.43 \mathrm{~Hz}$, which is about a cyclotron frequency of $\mathrm{SO}_{2}^{+}$near Io $(0.47 \mathrm{~Hz})$. Hence, described oscillations with frequency of $\sim 0.5 \mathrm{~Hz}$ have a good chance of being a modulation by ion cyclotron waves.

Although our analysis reveals some DAM modulation at the gyrofrequencies of the heaviest ions in the Io wake; however, this effect is episodical. A confirmation with more abundant experimental material is needed.

\section{Conclusions}

Besides the theoretical reasons, there are experimental arguments for the DAM modulation by propagating waves with ultralow frequencies:

(a) the regular patterns (moire) move with Alfvén velocity on the background of S-burst trains;

(b) rare whistler-like details are found in the dynamic power spectra of low frequency fluctuations of S-burst emission;

(c) the specific 2 s-modulation of S-burst emission corresponds to a frequency of about $0.5 \mathrm{~Hz}$ of the known magnetic pulsations near $f_{c i}$ of the heaviest ions in the Io torus $\left(\mathrm{SO}_{2}^{+}\right.$ and $\mathrm{SO}^{+}$).

It is very important that the parameters of modulating waves (a) and (b) allow estimation of the media parameters, which are in accordance with the results of other methods (space 
probe data, analysis of a standing wave in the ionospheric resonator). Hence, the revealed modulation effects seem to be real, although our results are a demonstrational precursor of future studies.

Whistlers and ion cyclotron waves are recorded in situ by space probes and used for plasma diagnostics. Hence, their modulations of DAM seem a valuable instrument for remote sensing of the Jovian magnetosphere.

Acknowledgements. We used the dynamical spectra of the S-storms recorded during an observation compaign within the framework of the INTAS project 0351-5727 (A. Lecacheux, H.O. Rucker, A.A. Konovalenko and Yu. Tokarev) with the UTR-2 radio telescope.

\section{References}

Arkhipov, A. V. 2003, Kin. Phys. Celest. Bod., 19, 265

Arkhipov, A. V. 2004, Kin. Phys. Celest. Bod., 20, 252

Arkhypov, O. V., \& Rucker, H. O. 2006, A\&A, 452, 347

Arkhypov, O. V., \& Rucker, H. O. 2007, A\&A, 467, 353

Ashcraft, T. 2000, Example of S-bursting during Io-B storm, http://www.jupiterradio.com/j/articles/ashcraft/ IoB101800_0807ut20MHzASH.wav

Bagenal, F., \& Leblanc, Y. 1988, A\&A, 197, 311

Belcher, J. W. 1987, Science, 238, 170

Borucki, W. J., Bar-Nun, A., Scarf, F. L., Cook, A. F., \& Hunt, G. E. 1982, Icarus, 52,492

Connerney, J. E. P., Acuna, M. H., Ness, N. F., \& Satoh, T. 1998, J. Geoph. Res., 103, A6, 11929

Ellis, G. R. A. 1982, Aust. J. Phys., 35, 165

Ergun, R. E., Su, Y.-J., Andersson, L., et al. 2006, J. Geoph. Res., 111, A6, doi:10.1029/2005JA011253

Farrell, W. M., Kaiser, M. L., \& Desch, M. D. 1999, Geophys. Res. Lett., 26, 16, 2601
Genova, F., \& Leblanc, Y. 1981, A\&A, 98, 133

Genova, F., Aubier, H. G., \& Lecacheux, A. 1981, A\&A, 104, 229

Gurnett, D. A., Shaw, R. R., Anderson, R. R., \& Kurth, W. S. 1979, Geophys. Res. Lett., 511

Gurnett, D. A., Scarf, F. L., Kurth, W. S., Shaw, R. R., \& Poynter, R. L. 1981, J. Geophys. Res., 86, A10, 8199

Hanasz, J., de Feraudy, H., Schreiber, R., \& Panchenko, M. 2006, J. Geophys. Res., 111, A03209, doi: 10.1029/2005JA011302

Hess, S., Zarka, P., \& Mottez, F. 2007, Planet. Space Sci., 55, 89

Imai, K., Wang, L., \& Carr, T. D. 1997, J. Geophys. Res., 102, A4, 7127

Kivelson, M. G., Khurana, K. K., Walker, R. J., et al. 1996, Science, 274, 396

Kuzmichev, V. E. 1989, The laws and formulas of physics (Kyiv: Naukova dumka), 394

Lecacheux, A., Konovalenko, A. A., \& Rucker, H. O. 2004, Planet. Space Sci., 52,1357

Mutel, R. L., Peterson, W. M., Jaeger, T. R., \& Scudder, J. D. 2007, J. Geophys. Res. 112, A7, doi: 10.1029/2007JA012442

Nicholson, D. R. 1983, Introduction to Plasma Theory (N.Y.: J. Wiley \& Sons), 140

Riihimaa, J. J. 1991, Earth, Moon and Planets, 53, 2, 157

Riihimaa, J. J. 1993, Modulation lanes revisited (Oulu: Univ. Oulu)

Russell, C. T., Blanco-Cano, X., Wang, Y. L., \& Kivelson, M. G. 2003, Planet. Space Sci., 51, 937

Strobel, D. F., \& Atreya, S. K. 1983, Ionosphere, in Physics of the Jovian magnetosphere, ed. A. J. Dessler (Cambridge: Cambridge University Press), 51 (Fig. 2.4)

Su, Y.-J., Jones, S. T., Ergun, R. E., et al. 2006, J. Geophys. Res. 111, A6, doi:10.1029/2005JA011252

Xin, L., \& Menietti, J. D. 2007, J. Geophys. Res. 112, A4, doi: 10.1029/2005JA011252

Yelle, R. V., \& Miller, S. 2004, Jupiters Thermosphere and Ionosphere, in Jupiter: the Planet, Satellites and Magnetosphere, ed. F. Bagenal, T. E. Dowling , \& W. B. McKinnon (Cambridge: Cambridge University Press), 185

Zarka, P. 1998, J. Geohys. Res., 103, E9, 20159 